\title{
INFARED IMAGING TECHNOLOGY IN THE FIELD OF STEEL INDUSTRY AND BUILDING
}

\author{
Lee han sung ${ }^{1 \dagger}$, Kwon hyuk il ${ }^{1}$ \\ ${ }^{1+}$ POSMATE, 504, Teheran ro, Gang nam gu, Seoul, Rep. KOREA \\ ${ }^{1}$ POSMATE, 504, Teheran ro, Gang nam gu, Seoul, Rep. KOREA \\ ${ }^{1 \dagger}$ Presenting Author : zip1212@posmate.com \\ 1Corresponding Author : kwonhil@posmate.com
}

\begin{abstract}
All objects above absolute zero $\left(-273{ }^{\circ} \mathrm{C}\right)$ emit energy in thermal radiation form. This release of energy can be detected using infrared sensors, which were first applied in 1989 by infrared camera technology to measure the temperature of the surface of the object. Since then, infrared imaging cameras have become widely used in various industries.

In this announcement, we present an overview of how infrared imaging cameras are applied in an industrial field. After reviewing cases of POSCO group and large sized office building, we were able to confirm that infrared imaging camera can be effective solution for equipment diagnosis.
\end{abstract}

KEYWORDS : Infrared thermal imaging, Diagnosis, Steel industry, Building

\section{INTRODUCTION}

With the upgrading of industry, facilities used on-site are increasingly operating at high voltages and high temperatures. Therefore, advanced technical skills and safety are required during the equipment diagnosis and inspection process.

In these sites, availability of infrared imaging camera is gradually increasing by measuring the surface temperature of equipment surface at a distance.

Infrared imaging camera is one of the best alternatives for diagnosing facilities in extreme environments that have been difficult to measure since it is careful and non-contact method.

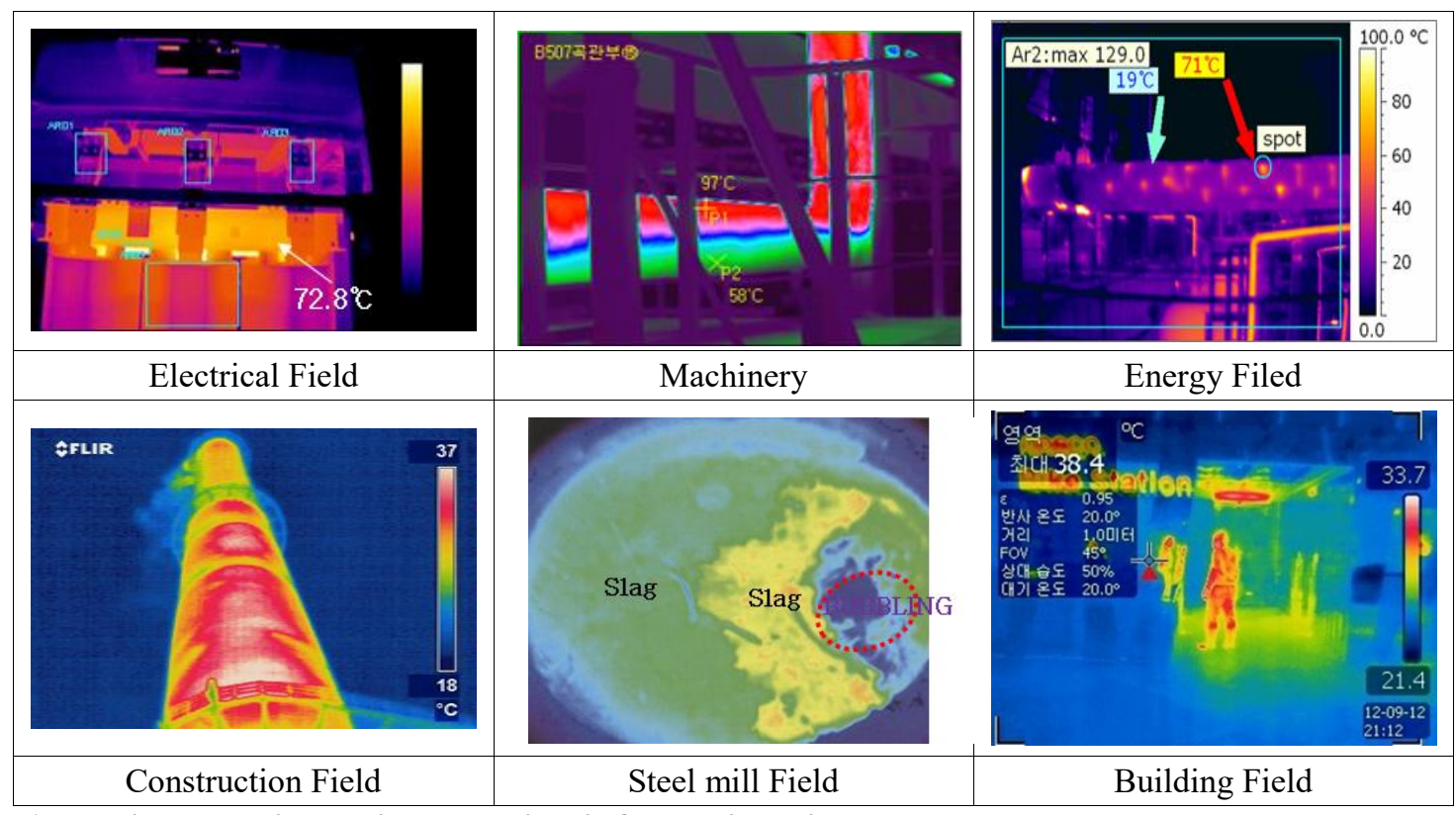

Figure 1. Equipment diagnosis case using infrared imaging camera 


\section{APPLICATION CASES}

\subsection{Electrical field}

The flow of current at conductor generates joule's heat. This joule's heat cause excessive heat wave and fire. To prevent these accidents, we can previously diagnose equipment with infrared imaging camera. The examples are electrical cable tray, power line tower, circuit breaker, motor and PCB.

\subsection{Machinery}

The temperature of pipes, structure transferring fluid or solid material is partially lowered when clogging of the conveyed matter occurs inside. This can be diagnosed by infrared imaging camera and finding them without physically damaging the transport track.

\subsection{Energy filed}

It is possible to find a place where steam used as a heat source for heating and cooling the building loses and to diagnose a location where heat insulation is poor by using infrared camera. This can reduce the heat loss of the building and improve the energy waste that occur continuously.

\subsection{Construction Field}

When there is a temperature difference between the inside and outside of the structure, a larger temperature difference is exhibited in a portion where the thickness of the structure is thin and the heat insulation is low. In case of aging stack structure, the more it is damaged, the higher the temperature becomes. This can be diagnosed and a plan for replacement of buildings can be established. It can also be used in performance tests of insulating coatings to predict the insulation performance prior to application.

\subsection{Steel mill field}

A thermal imaging camera can be installed on the top of the ladle, which is a container for receiving and transporting molten metal, to monitor the bubbling phenomenon of molten metal. The bubbling phenomenon affects the quality of steel, but it can only be monitored by using an infrared camera because it can lead to visual damage to the operator if the molten metal is seen with the naked eye.

\subsection{Building field}

Infrared imaging cameras are used for most facilities such as power transform facilities, boilers, and construction materials in buildings. For example, there is a case in which the heat generated by the defective fastening bolts in high voltage bus bar and the overheating inside the electric panel are detected. In addition, it can be used for the detection of suspicious person in the night that can't be done by CCTV, and the detection of pyrogenic disease patients.

\section{CONCLUSION}

In the early days, infrared thermal cameras have been used only for limited use in the industrial field, but in recent years, the use of the infrared imaging cameras has been increasing due to the miniaturization, multi-functioning and accumulation of user experience. Accordingly, it has been widely used in the private sectors as well as in the security. Also it is been used in sanitation fields of general buildings beyond the scope of diagnosis of industrial facilities. As shown in the previous examples, infrared imaging technology is likely to be applied to various fields due to its non-contact, portability, and precision features.

\section{REFERENCES}

[1] Kwon Hyuk Il, Infrared thermal imaging technology in the field of steel industry and building, 2016 